OPEN ACCESS

Edited by:

Shiv K. Gupta,

Mayo Clinic, United States

Reviewed by:

Sharanjot+ Saini,

University of California, San Francisco,

United States

Mihnea P. Dragomir,

Charité University Medicine Berlin,

Germany

Dongya Zhang

Nanjing University of Chinese

Medicine, China

*Correspondence: Ana Laura Pereira-Suárez analauraps@hotmail.com

Specialty section:

This article was submitted to Molecular and Cellular Oncology,

a section of the journal

Frontiers in Cell and Developmental

Biology

Received: 31 May 2021

Accepted: 31 October 2021

Published: 29 November 2021

Citation:

Villegas-Pineda JC

Lizarazo-Taborda MdR,

Ramírez-de-Arellano $A$ and

Pereira-Suárez AL (2021) Exosomal miRNAs and IncRNAs: The Modulator

Keys of Cancer-Associated

Fibroblasts in the Genesis and

Progression of Malignant Neoplasms.

Front. Cell Dev. Biol. 9:717478.

doi: $10.3389 /$ fcell.2021.717478

\section{Exosomal miRNAs and IncRNAs: The Modulator Keys of Cancer-Associated Fibroblasts in the Genesis and Progression of Malignant Neoplasms}

\author{
Julio César Villegas-Pineda ${ }^{1,2}$, Mélida del Rosario Lizarazo-Taborda ${ }^{3}$, \\ Adrián Ramírez-de-Arellano ${ }^{2}$ and Ana Laura Pereira-Suárez ${ }^{2,4 *}$
}

${ }^{1}$ Doctorado en Ciencias Biomédicas, Departamento de Fisiología, Centro Universitario de Ciencias de la Salud, Universidad de Guadalajara, Guadalajara, Mexico, ${ }^{2}$ Instituto de Investigación en Ciencias Biomédicas, Centro Universitario de Ciencias de la Salud, Universidad de Guadalajara, Guadalajara, Mexico, ${ }^{3}$ Programa de Bacteriología y Laboratorio Clínico, Facultad de Ciencias de la Salud, Universidad de Santander, Cúcuta, Colombia, ${ }^{4}$ Departamento de Microbiología y Patología, Centro Universitario de Ciencias de la Salud, Universidad de Guadalajara, Guadalajara, Mexico

The tumor microenvironment is made up of a universe of molecular and cellular components that promote or inhibit the development of neoplasms. Among the molecular elements are cytokines, metalloproteinases, proteins, mitochondrial DNA, and nucleic acids, within which the ncRNAs: miRNAs and IncRNAs stand out due to their direct modulating effects on the genesis and progression of various cancers. Regarding cellular elements, the solid tumor microenvironment is made up of tumor cells, healthy adjacent epithelial cells, immune system cells, endothelial cells, and stromal cells, such as cancer-associated fibroblasts, which are capable of generating a modulating communication network with the other components of the tumor microenvironment through, among other mechanisms, the secretion of exosomal vesicles loaded with miRNAs and IncRNAs. These ncRNAs are key pieces in developing neoplasms since they have diverse effects on cancer cells and healthy cells, favoring or negatively regulating protumoral cellular events, such as migration, invasion, proliferation, metastasis, epithelial-mesenchymal transition, and resistance to treatment. Due to the growing number of relevant evidence in recent years, this work focused on reviewing, analyzing, highlighting, and showing the current state of research on exosomal ncRNAs derived from cancer-associated fibroblasts and their effects on different neoplasms. A future perspective on using these ncRNAs as real therapeutic tools in the treatment of cancer patients is also proposed.

Keywords: cancer, cancer-associated fibroblast, tumor microenvironment, exosomes, miRNAs, IncRNAs

\section{INTRODUCTION}

Malignant tumors consist of cancer cells and tumor-associated host cells (De Wever et al., 2014), the interaction between tumor microenvironment (TME) and tumor cells plays a key role in cancer progression (Dayan et al., 2012; Ali et al., 2015; Shah et al., 2015; Vered et al., 2015; Ringuette Goulet et al., 2018; Zhang Y.-F. et al., 2019; Dou et al., 2020; Wu et al., 2020). Remarkably, the dynamic interplay between cancer-associated fibroblasts (CAFs), cancer cells, and healthy cells has an essential role during tumor initiation and growth (Zhao et al., 2016; Li K. et al., 2020; Lv et al., 2020). In recent years, exosomes have gained relevance due to their regulatory role in the carcinogenesis of different neoplasms (Nilsson 


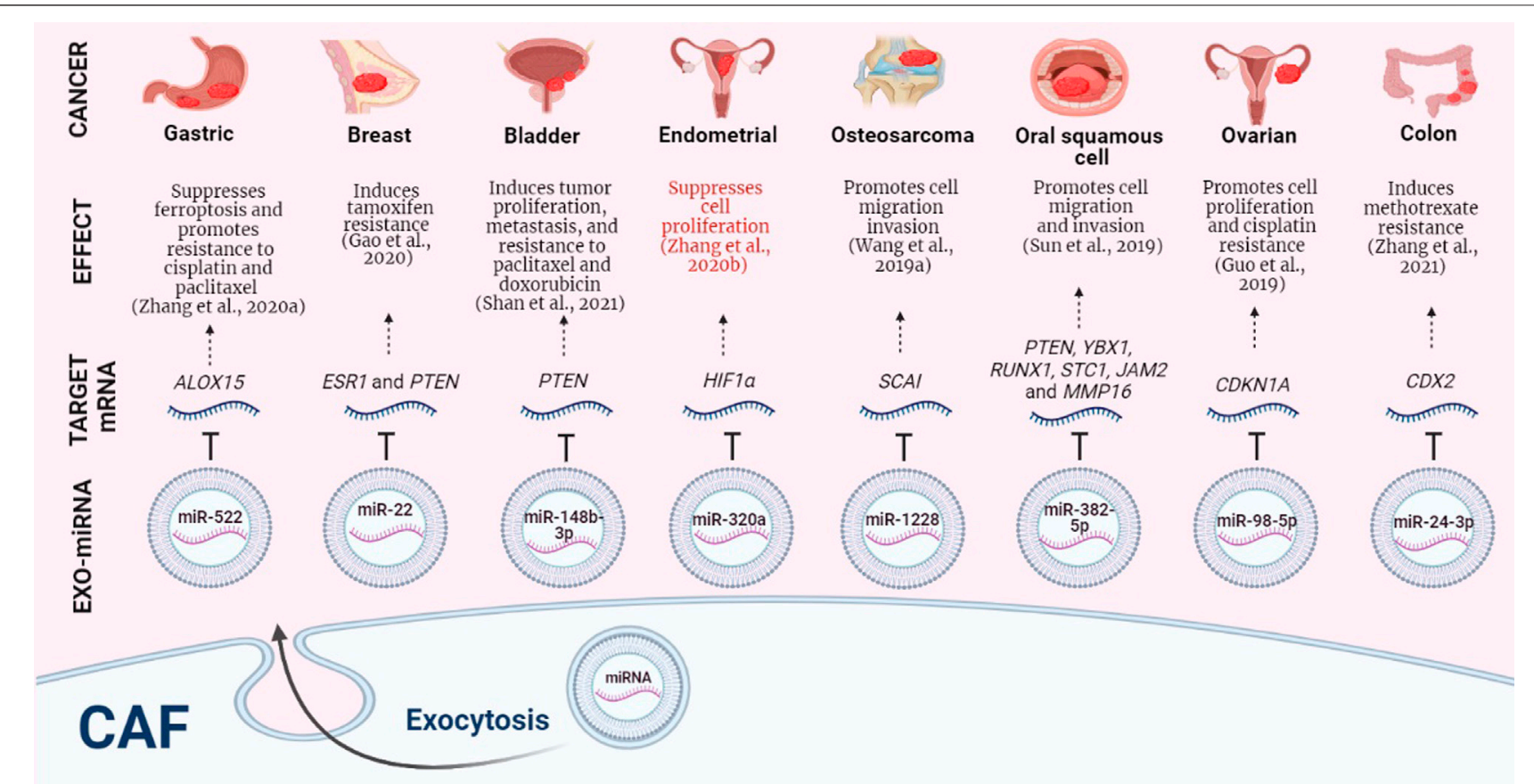

FIGURE 1 | CAFs-derived exosomal miRNAs, their target mRNAs and effects on different neoplasms. Protumoral events in black, antitumor events in red. CAF: Cancer-associated fibroblast. EXO-miRNA: exosomal miRNA. Image created in BioRender.com.

et al., 2009; Ramteke et al., 2015; Donnarumma et al., 2017; Seo et al., 2018; Zhang Y. et al., 2019; Lee et al., 2020). Exosomes are MHC class I- and class II-bearing nanovesicles of endocytic origin; their size is in the range of 30-100 nm (Admyre et al., 2007; Nilsson et al., 2009). Exosomes can participate in intercellular communication between cells that make up the TME by transmitting intracellular cargoes (Achreja et al., 2017; Qin et al., 2019), their content can be miscellaneous and include macromolecules such as cytokines (Mashouri et al., 2019), metalloproteinases (Shimoda et al., 2014), proteins (Mathivanan et al., 2010; Zhang Y.-F. et al., 2019; Zhao et al., 2020), mitochondrial DNA (mtDNA) (Sansone et al., 2017) and can be enriched with different types of RNAs, such as messenger RNAs (mRNAs) (Gener Lahav et al., 2019), microRNAs [miRNAs or miR-, single-stranded non-coding RNAs (ncRNAs) of 20 nucleotides in length that are endogenously expressed (Beermann et al., 2016)] (Théry, 2011; Nouraee et al., 2016; Qin et al., 2019), about this type of ncRNAs, Dragomir et al., in their work entitled "SnapShot: Unconventional miRNA Functions," mention that miRNAs can regulate gene expression at the post-transcriptional level in a conventional way by binding to mRNAs, resulting in the disintegration of target mRNAs and inhibition of translation, additionally, the authors also highlight in an exceptional way that through various mechanisms, such as the activation of Toll-like receptors, the upregulation of protein expression, the targeting of mitochondrial transcripts, the direct activation of transcription, among others, miRNAs can carry out their regulatory functions in an unconventional way (Dragomir et al., 2018), and long non-coding RNAs (lncRNAs, transcripts that are longer than 200 nucleotides and do not harbor protein-coding signatures (Beermann et al., 2016)) (Zhou et al., 2021). Exosomes can be produced by immune system cells (Elashiry et al., 2021), epithelial cells (Han et al., 2017), tumor cells (Gu et al., 2012; Cheng et al., 2017; Ding et al., 2018; Chen et al., 2019; Hu and Hu, 2019; Amit et al., 2020; Jung et al., 2020; Yang et al., 2020) and CAFs (Herrera et al., 2018; Principe et al., 2018; Liu et al., 2020). CAFs are resident stromal cells of the TME; they have a promoting effect on carcinogenesis and progression of different neoplasms (Luga and Wrana, 2013; Huang et al., 2019; Sun and $\mathrm{Fu}, 2019)$ by transferring exosomes carrying ncRNAs to cells of the TME (Richards et al., 2017; Fiori et al., 2019) (Figure 1).

For everything mentioned above, the present work is based on the recent findings on the protumoral and antitumoral effects of CAFsderived exosomal miRNAs and lncRNAs; it focuses on analyzing and highlighting the importance of these ncRNAs in the genesis and progression of different cancers. At the same time, it shows the current situation of this new growing research area, and a future perspective on the use of these ncRNAs as real therapeutic tools in the treatment of cancer patients is also proposed.

\section{EXOSOMES: INDUCING FACTOR IN THE FORMATION OF CAFS AND TUMOR MICROENVIRONMENT MODULATOR}

Exosomes are among the most recognized inducers for generating CAFs (Li K. et al., 2020). CAFs are known as cells capable of secreting exosomes (You et al., 2019), and in turn, exosomes derived from cancer cells containing TGF- $\beta$ can induce the generation of CAFs from stromal cells (Goulet et al., 2019). It has also been observed that exosomes derived from CAFs containing TGF- $\beta$ activate the SMAD signaling pathway in cancer cells through a particular type of 
epithelial-mesenchymal transition (EMT), which increases their malignant behavior (Li et al., 2017), thus generating a loop between CAFs and exosomes. It has been shown that chronic lymphocytic leukemia cells-derived exosomes can transfer miR146a to bone marrow-derived mesenchymal stem cells to generate CAFs; this is done by promoting EMT by targeting ubiquitin specific peptidase 16 (USP16) (Yang et al., 2020). Another cellular transition process in which exosomes are involved to originate CAFs is the endothelial-mesenchymal transition; this event was demonstrated by observing that melanoma-derived exosomes loaded with TGF- $\beta$ induced the transition from human umbilical vein endothelial cells (HUVECs) to differentiated CAFs (Yeon et al., 2018). For their part, Baroni et al. demonstrated that triple-negative breast CAFs-derived exosomal miR-9 could generate a cell type with CAF-like properties in human breast fibroblasts, in which this miRNA enhanced the capacity for migration and invasion, as in breast cancer cell lines. Additionally, overexpression of this ncRNA in normal fibroblasts was able to promote tumor growth in a murine orthotopic xenograft model (Baroni et al., 2016).

It has also been observed that the exosomes produced by CAFs can modulate TME. For example, miR-21, which is packed in CAFderived exosomes, induces the generation of monocytic myeloidderived suppressor cells by activating STAT3 (Zhao et al., 2021). On a breast cancer model, exosomes produced by CAFs can also interfere with immunologic processes. In breast cancer cells treated with exosomes derived from CAFs, an increase of miR-92 was observed, which was essential for migration and invasion and correlated with the suppression of the immune cell function and the promotion of PD-L1 expression in these cells (Dou et al., 2020). Also, in the metastatic lung niche, a higher capacity to induce the transformation fibroblast into CAFs has been observed due to exosomal transport of miR-1247-3p from high-metastatic hepatocellular carcinoma cells; leading to a pro-inflammatory microenvironment promoted by CAFs, which secret IL-6 and IL8, among other cytokines (Fang et al., 2018).

Considering this evidence, the important reciprocal relationship between exosomes and CAFs in the latter's self-generation and consequently in neoplasms' development is manifest. This relationship could be explored in greater depth and considered a previous or potentiating stage of carcinogenesis to inhibit the exosomal release and block exosomal molecules essential for the initiation, establishment, and progression of different cancers.

\section{CELLULAR EFFECTS OF CAFS-DERIVED EXOSOMAL MIRNAS AND LNCRNAS ON KEY EVENTS FOR GENESIS AND PROGRESSION OF NEOPLASMS}

\section{Migration and Invasion, Processes Upregulated by CAFs-Derived Exosomal ncRNAs}

Migration and invasion of cancer cells are essential for the establishment of the malignant neoplasm. Sun et al. isolated CAFs from patients with oral squamous cell carcinoma
(OSCC) and showed that in tongue squamous cell carcinoma CAL-27 cells, CAFs could promote these two events through the transfer of exosomal miR-382-5p, which facilitated the OSCC progression. Despite not having biologically determined the interaction, an analysis in silico predicted PTEN, YBX1, RUNX1, STC1, JAM2, and MMP16 as candidate target genes for this miRNA (Sun et al., 2019). Another exosomal miRNA that has been shown to promote cell migration and invasion is miR1228; this small non-coding regulatory RNA was enriched in exosomes secreted by CAFs and could downregulate endogenous SCAI mRNA and protein level in osteosarcoma, contributing to carcinogenesis of this neoplasm (Wang J.-W. et al., 2019).

CAFs-derived exosomes can also carry lncRNAs; LINC00659 is an example of this; it was found enriched in CAFs-derived exosomes and was shown to have multiple effects on human colorectal cancer (CRC) cells, it was able to induce cell proliferation, migration, invasion, and EMT in vitro. Furthermore, it was determined that these events were promoted by directly interacting with the tumor suppressor miR-342-3p to increase ANXA2 expression in CRC cells (Zhou et al., 2021).

The protumoral effects exerted by various CAFs-derived exosomal miRNAs and lncRNAs enhance the migration and invasion of cancer cells and convert them into key pieces within the universe of participants in developing neoplasms.

\section{CAFs-Derived Exosomal ncRNAs Promote Proliferation and Metastasis, Essential Malignant Characteristics for Tumor Progression}

Among the multiple protumoral effects that CAFs-derived exosomal ncRNAs exert on cancer cells are the promotion of cell proliferation and metastasis. Chen et al. determined that CAFs isolated from samples of patients with invasive ductal carcinoma were capable of transferring exosomal miR-500a-5p to breast cancer cell lines, this miRNA bound to ubiquitin-specific peptidase 28 (USP28) promoting cell proliferation and metastasis in a nude mouse xenograft model (Chen et al., 2021). It has also been shown that miR-181d-5p can promote various protumoral cellular events in breast cancer cells, such as proliferation, invasion, and migration. If not enough, it can also induce EMT, antagonize apoptosis in vitro of breast cancer cells and promote tumor growth in nude mice xenografted with MCF-7 cells via downregulation of the transcription factors CDX2 and HOXA5 (Wang et al., 2020).

Another important effect carried out by CAFs exosomal ncRNAs is the reprogramming of metabolic pathways. Although to date, this aspect has not yet been studied in-depth, there is a report where a protumoral role is proposed for lncRNA SNHG3, which positively regulated pyruvate kinase isozymes M1/M2 (PKM) expression, inhibited mitochondrial oxidative phosphorylation, increased glycolysis, and proliferation of breast tumor cells through a mechanism similar to a molecular sponge for antitumoral miR330-5p (Li Y. et al., 2020); this highlights the multifunctionality that CAFs-derived exosomal ncRNAs can exert on various processes related to carcinogenesis and tumor progression. 
Considering the evidence reported, CAFs-derived exosomal miRNAs and lncRNAs can participate directly in tumor growth and the dissemination of cancer cells to other anatomical sites adjacent or far from the primary site, which could cause functional compromise of multiple organs and consequent serious complications in cancer patients.

\section{Treatment Resistance Generated by CAFs-Derived Exosomal miRNAs and IncRNAs}

CAFs are an important factor in generating tumor resistance to treatment (Fiori et al., 2019); this protumoral feature is associated with worsening cancer patients' prognosis (Domvri et al., 2020; Huang et al., 2020). CAFs possess chemoresistance by innate nature and, it has been observed that in the presence of gemcitabine, a cytotoxic anticancer chemotherapy drug, they can transfer this characteristic to cancer cells. CAFs exposed to this antineoplastic drug could increase the release of miR-146aloaded exosomes; in in vitro assays, this miRNA transmitted gemcitabine resistance to pancreatic cancer epithelial cell lines, promoting cell proliferation and survival. In the same work, it was shown that miR-146a is directly regulated by the promoter binding transcription factor, Snail, which acts as a chemoresistance-inducing factor, and that it was also found to be overexpressed in human pancreatic CAFs-derived exosomes (Richards et al., 2017). Recently, Gao et al. identified and characterized a subtype of CAFs which particularly has the presence of the cell surface protein CD63; these $\mathrm{CD}^{+}{ }^{+} \mathrm{CAF}$ are capable of secreting exosomes enriched in miR-22, which can bind to their targets, ER $\alpha$ and PTEN, and deregulate their expression, thus conferring tamoxifen resistance to breast cancer cells (Gao et al., 2020).

Gemcitabine and tamoxifen are not the only drugs for which CAFs-derived exosomal miRNAs generate resistance in cancer cells; in fact, a considerable number of reports show that the cytotoxic effect caused by cisplatin in cancer cells can be diminished or eliminated by the action of CAFs-derived exosomal ncRNAs. It has been shown that, in cisplatin-sensitive ovarian cancer cells, CAFs-derived exosomal miR-98-5p was capable of binding to cyclin-dependent kinase inhibitor 1A (CDKN1A) to inhibit its expression, which increased ovarian cancer cell proliferation and cell cycle entry, suppressed cell apoptosis, and promoted cisplatin resistance in vitro and in a xenotransplanted nude mouse model (Guo et al., 2019). The intrinsic resistance that CAFs have towards cisplatin also favors the progression of head and neck cancer (HNC). It has been observed that CAFs can transfer exosomal miR-196a to HNC cells, which generated cell survival, proliferation, and inhibition of apoptosis, also conferred cisplatin resistance by targeting CDKN1B and ING5, cell cycle inhibitor and tumor suppressor molecules, respectively. Additionally, high levels of exosomal miR-196a in plasma were clinically correlated with poor overall survival and chemoresistance in patients with HNC (Qin et al., 2019).

There is a great diversity between the protumoral effects generated by CAFs-derived exosomal miRNAs and IncRNAs; it has been shown that miR-522 is capable of inhibiting ferroptosis, a novel mode of non-apoptotic cell death induced by a build-up of toxic lipid peroxides (lipid-ROS) in an irondependent manner, in gastric cancer cells by targeting ALOX15 and blocking lipid-ROS accumulation. Additionally, assays performed in an orthotopic implantation model in nude mice to evaluate gastric tumor growth and chemosensitivity led the authors to suggest that CAFs-derived exosomes containing miR522 promote a new mechanism of acquired chemoresistance to cisplatin through an intercellular pathway, comprising USP7, hnRNPA1, miR-522, and ALOX15 (Zhang H. et al., 2020).

On the other hand, it has been observed that miR-423-5ploaded exosomes derived from CAFs can decrease the chemosensitivity of prostate cancer cells and increase the resistance of cells resistant to taxanes; in addition, it was shown that the inhibition of this miRNA enhanced the drug sensitivity of prostate cancer cells in a tumor xenograft model in nude mice. These protumoral events were favored due to the inhibitory effect of miR-423-5p on GREM2 and the impact exerted on the TGF- $\beta$ pathway (Shan et al., 2020). In another type of neoplasm, in bladder cancer, it was found that miR-148b$3 \mathrm{p}$ can also induce resistance to treatment. Shan et al. validated PTEN as a target of miR-148b-3p; the negative dysregulation of PTEN promoted metastasis, EMT, and resistance to doxorubicin and paclitaxel both in in vitro assays using bladder cancer cells and in in vivo assays using a xenograft mouse model. These events were generated due to the tumor-promoting effects of miR-148b3 p via the Wnt/ $\beta$-catenin pathway (Shan et al., 2021).

CAFs-derived exosomal miRNAs have also shown an inhibitory effect on the cytotoxic action that methotrexate exerts on the metabolism of colon cancer cells. In an in vivo model of colon cancer, it was shown that miR-24-3p induced resistance to this drug, favoring tumor growth under treatment of methotrexate by down-regulating the $\mathrm{CDX} 2 / \mathrm{HEPH}$ axis (Zhang et al., 2021).

As mentioned previously, CAFs are also capable of secreting exosomal lncRNAs that promote tumor progression. Such is the case of exosomal lncUCA1, which conferred resistance to cisplatin in vulvar squamous cell carcinoma cells. Mechanistically, lncUCA1 functioned as a sponge for miR103a, a miRNA with antitumoral function in various human cancers, this sequestration of miR-103a by lncUCA1 promoted the expression of WEE1 G2 checkpoint kinase (WEE1), a direct target of miR-103a, enhancing tumor growth and cisplatin resistance in a $\mathrm{BALB} / \mathrm{c}$ nude xenograft model (Gao et al., 2021). Another CAFs-derived exosomal lncRNA with protumoral effect is H19, highly expressed in tumors generated in an azoxymethane (AOM)/dextran sodium sulfate (DSS) model of colitis-associated cancer, as well as in CRC samples from patients in different tumor-node-metastasis stages. Additionally, this lncRNA promoted the stemness of CRC stem cells, increased the frequency of tumor-initiating cells, and promoted the resistance of CRC cells to oxaliplatin both in vitro and in vivo. Mechanistically, H19 activated the $\beta$-catenin pathway by acting as an endogenous competitor for miR-141, a miRNA with antitumoral effect, in CRC cells (Ren et al., 2018).

These findings propose miRNAs and lncRNAs as important obstacles to achieving a successful chemotherapeutic treatment; 
TABLE 1 | Cellular and tumor effects and mechanisms exerted by exosomal miRNAs and IncRNAs derived from CAFs in various cancers.

\begin{tabular}{|c|c|c|c|c|}
\hline $\begin{array}{l}\text { Exosomal } \\
\text { ncRNAs } \\
\text { Tumor effect }\end{array}$ & Cellular effects & Mechanisms & Cancer & References \\
\hline $\begin{array}{l}\text { miR-92 } \\
\text { PRO-TUMOR }\end{array}$ & $\begin{array}{l}\text { Promotes apoptosis and impairs proliferation of T cells, } \\
\text { increases proliferation and migration of breast cancer } \\
\text { cells, and facilitates tumor progression }\end{array}$ & $\begin{array}{l}\text { Targets LATS2 and modulates the LATS2-YAP1 axis } \\
\text { generating an incremented expression of PD-L1 }\end{array}$ & Breast & $\begin{array}{l}\text { Dou et al. } \\
(2020)\end{array}$ \\
\hline $\begin{array}{l}\text { miR-196a } \\
\text { PRO-TUMOR }\end{array}$ & $\begin{array}{l}\text { Promotes cell proliferation and confers cisplatin } \\
\text { resistance }\end{array}$ & Targets and downregulates CDKN1B and ING5 & Head and neck & Qin et al. (2019) \\
\hline $\begin{array}{l}\text { IncRNA } \\
\text { LINC00659 } \\
\text { PRO-TUMOR }\end{array}$ & $\begin{array}{l}\text { Promotes cell proliferation, migration, invasion, and EMT } \\
\text { progression }\end{array}$ & $\begin{array}{l}\text { Interacts directly with miR-342-3p to increase ANXA2 } \\
\text { expression }\end{array}$ & Colorectal & $\begin{array}{l}\text { Zhou et al. } \\
\text { (2021) }\end{array}$ \\
\hline $\begin{array}{l}\text { IncRNA-CAF } \\
\text { PRO-TUMOR }\end{array}$ & Increases cell proliferation and promotes tumor growth & $\begin{array}{l}\text { Stabilizes and up-regulates cytokine IL-33 to reprogram } \\
\text { CAFs }\end{array}$ & $\begin{array}{l}\text { Oral squamous } \\
\text { cell }\end{array}$ & $\begin{array}{l}\text { Ding et al. } \\
(2018)\end{array}$ \\
\hline $\begin{array}{l}\text { miR-500a-5p } \\
\text { PRO-TUMOR }\end{array}$ & Enhances cell proliferation and induces metastasis & $\begin{array}{l}\text { Binds to tumor suppressor ubiquitin-specific peptidase } \\
28 \text { (USP28) }\end{array}$ & Breast & $\begin{array}{l}\text { Chen et al. } \\
(2021)\end{array}$ \\
\hline $\begin{array}{l}\text { miR-181d-5p } \\
\text { PRO-TUMOR }\end{array}$ & $\begin{array}{l}\text { Induces cell proliferation, invasion, migration, and EMT, } \\
\text { antagonizes apoptosis in vitro, and promotes tumor } \\
\text { growth in vivo }\end{array}$ & $\begin{array}{l}\text { Downregulates the expression of the transcription } \\
\text { factors CDX2 and HOXA5 }\end{array}$ & Breast & $\begin{array}{l}\text { Wang et al. } \\
(2020)\end{array}$ \\
\hline $\begin{array}{l}\text { miR-423-5p } \\
\text { PRO-TUMOR }\end{array}$ & Promotes chemotherapy resistance & $\begin{array}{l}\text { Targets GREM } 2 \text { to inhibit its expression and favors the } \\
\text { TGF- } \beta \text { pathway }\end{array}$ & Prostate & $\begin{array}{l}\text { Shan et al. } \\
(2020)\end{array}$ \\
\hline $\begin{array}{l}\text { IncRNA UCA1 } \\
\text { PRO-TUMOR }\end{array}$ & Enhances tumor growth and chemoresistance & $\begin{array}{l}\text { Favors the expression of WEE1 through sponging miR- } \\
103 a\end{array}$ & $\begin{array}{l}\text { Vulvar } \\
\text { squamous cell }\end{array}$ & $\begin{array}{l}\text { Gao et al. } \\
(2021)\end{array}$ \\
\hline $\begin{array}{l}\text { IncRNA H19 } \\
\text { PRO-TUMOR }\end{array}$ & Promotes stemness and chemoresistance & $\begin{array}{l}\text { Activates the } \beta \text {-catenin pathway, acting as a competing } \\
\text { endogenous RNA sponge for miR-141 }\end{array}$ & Colorectal & $\begin{array}{l}\text { Ren et al. } \\
\text { (2018) }\end{array}$ \\
\hline $\begin{array}{l}\text { IncRNA SNHG3 } \\
\text { PRO-TUMOR }\end{array}$ & $\begin{array}{l}\text { Inhibits mitochondrial oxidative phosphorylation, } \\
\text { increases glycolysis, and enhances cell proliferation }\end{array}$ & $\begin{array}{l}\text { Functions as a molecular sponge of miR-330-5p to } \\
\text { regulate the expression of PKM }\end{array}$ & Breast & Li et al. (2020b) \\
\hline $\begin{array}{l}\text { miR-148b } \\
\text { ANTI-TUMOR }\end{array}$ & Inhibits EMT and reduces cell invasion and metastasis & Directly binds to DNMT1 and decreases MMP-9 activity & Endometrial & Li et al. (2019) \\
\hline $\begin{array}{l}\text { miR-4516 } \\
\text { ANTI-TUMOR }\end{array}$ & Suppresses cell proliferation & Targets FOSL1, proliferation-related gene & Breast & $\begin{array}{l}\text { Kim et al. } \\
(2020)\end{array}$ \\
\hline $\begin{array}{l}\text { miR-320a } \\
\text { ANTI-TUMOR }\end{array}$ & $\begin{array}{l}\text { Inhibits EMT, cell proliferation, migration, invasion, } \\
\text { tumorigenesis, and metastasis }\end{array}$ & $\begin{array}{l}\text { Binds to } P B X 3 \text {, suppresses the activation of the MAPK } \\
\text { pathway, affects the expression of CDK2, and MMP2 } \\
\text { proteins due to reduced phosphorylation of ERK1/2 }\end{array}$ & Hepatocellular & $\begin{array}{l}\text { Zhang et al. } \\
(2017)\end{array}$ \\
\hline $\begin{array}{l}\text { miR-3188 } \\
\text { ANTI-TUMOR }\end{array}$ & $\begin{array}{l}\text { Reduces cell proliferation, promotes apoptosis, and } \\
\text { inhibits tumor growth }\end{array}$ & Directly targets $B C L 2$ & Head and neck & $\begin{array}{l}\text { Wang et al. } \\
\text { (2019b) }\end{array}$ \\
\hline $\begin{array}{l}\text { miR-34a-5p } \\
\text { ANTI-TUMOR }\end{array}$ & $\begin{array}{l}\text { Inhibits cell proliferation, migration, invasion, EMT, and } \\
\text { tumorigenesis }\end{array}$ & $\begin{array}{l}\text { Targets } A X L \text {, regulates the AKT/GSK- } 3 \beta / \beta \text {-catenin/ } \\
\text { Snail signaling cascade, and inhibits MMP- } 2 / 9\end{array}$ & Oral & Li et al. (2018) \\
\hline
\end{tabular}

subject to further studies confirming this evidence, the elimination or reduction of CAFs-derived exosomal ncRNAs that generate chemoresistance should be considered as a new oncological therapeutic strategy.

\section{Exosomal miRNAs With Antitumoral Effects} miRNAs have generally been reported as protumoral molecules; however, some reports suggest that their silencing or elimination could favor tumor development. A feature shared between the antitumoral miRNAs is that they are significantly reduced in CAFs-derived exosomes. In vitro and in vivo studies carried out by $\mathrm{Li}$ et al. revealed that miR-148b could function as a tumor suppressor, the downregulation of this miRNA induced EMT, migration, invasion, and increased MMP-9 activity in endometrial cancer cell lines, as well as metastasis in a nude mouse model, additionally, DNA methyltransferase 1 (DNMT1) was determined as the target gene of miR-148b (Li et al., 2019). This enzyme has also been associated with tumorigenesis in breast cancer due to its indispensable role in the maintenance of mammary stem/progenitor cell and cancer stem cell maintenance (Pathania et al., 2015), and chemoresistance to cisplatin in human non-small cell lung cancer cell lines (Sui et al., 2015). Another study also carried out in endometrial cancer showed that the CAFs-derived exosomal miR-320a inhibited the proliferation of cell lines by direct targeting and downregulation of hypoxia-inducible factor 1- $\alpha$ (HIF $\alpha$ ) (Zhang N. et al., 2020). This transcription factor has been strongly related to metastasis, angiogenesis, poor patient prognosis, and tumor resistance therapy (Masoud and Li, 2015). In another neoplasm typical of women, the CAFs-derived exosomal miR-4516 was isolated from tumor tissue obtained from a patient with invasive breast ductal carcinoma; this miRNA suppressed the proliferation of breast cancer cell lines by targeting FOSL1 (Kim et al., 2020).

The antitumoral effects that some miRNAs possess have not only been reported in gynecological cancers. In vitro and in vivo studies carried out in human hepatocellular carcinoma cell lines and in a nude mouse model, respectively, showed that miR-320a could function as an antitumoral miRNA, whose binding to $P B X 3$ affected the protein expression of cyclin-dependent kinase 2 (CDK2) and MMP-2 due to the reduction of the phosphorylation of ERK1/2. In this way, the miR-320a/PBX3 axis suppressed essential events for cancer progression such as EMT, cell proliferation, migration, invasion, tumorigenesis, and metastasis (Zhang et al., 2017). miR-3188 is another miRNA that 
has an antitumoral effect; its loss of CAFs-derived exosomes contributed to the malignancy of HNC cells, increasing cell proliferation, migration, and invasion, and inhibited apoptosis by derepressing its target B-cell lymphoma 2 (BCL2) mRNA, this miRNA was also able to inhibit tumor growth in a BALB/c nude mice model (Wang X. et al., 2019).

Another of the few reports in which evidence is presented about the antitumoral effect of miRNAs is made by Li et al. It was observed that the overexpression of CAFs-derived exosomal miR$34 a-5 p$ suppressed the tumorigenesis of OSCC cells in an immunodeficient $\mathrm{BALB} / \mathrm{c}$ mice subcutaneous tumor model. Additionally, in OSCC cell lines, it was shown that this miRNA is capable of binding directly to $A X L$, thus modulating the AKT/GSK-3 $\beta / \beta$-catenin signaling pathway; this event reduced proliferation, migration, and invasion, and decreased nuclear translocation of $\beta$-catenin, which led to decreased expression of Snail, a transcription factor of MMP-2 and MMP-9, important for EMT (Li et al., 2018).

Considering the reported evidence of some miRNAs' antitumoral effects in gynecological and non-gynecological cancers, these ncRNAs could become valuable tools to inhibit essential points in carcinogenesis and disease progression.

\section{CAFS-DERIVED EXOSOMAL MIRNAS AND LNCRNAS AS CANDIDATES FOR THERAPEUTIC TOOLS AND BIOMARKERS}

Given the importance of protumoral and antitumoral miRNAs and lncRNAs in processes such as cell proliferation, migration, invasion, EMT, metastasis, metabolism, resistance to treatment (Table 1), these exosomal ncRNAs derived from CAFs have been suggested as strong candidates that could be used as new targets or therapeutic tools in breast, ovarian, vulvar squamous cell, endometrial, head and neck, pancreas, oral squamous cell, gastric, bladder, colon, liver, prostate cancer, as well as biomarkers of clinical relevance; CAFs-derived exosomal ncRNAs can be considered cornerstones in neoplastic processes.

\section{CONCLUSION AND PERSPECTIVES}

Exosomes loaded with miRNAs and lncRNAs are a key communication pathway between CAFs and the different elements of the TME. Its relevance lies mainly in the promoter effect of protumoral and antitumoral events that modulate the genesis and the progression of various neoplasms. In the present work, the conventional effect of two types of exosomal ncRNAs derived from CAFs: miRNAs and lncRNAs, has been reviewed; this effect inhibits the translation by binding to its target mRNAs (Dragomir et al., 2018). Considering that cancer cells need different stimuli to form tumors and that CAFs, through the secretion of exosomal ncRNAs, among other mechanisms, can contribute significantly by favoring protumoral events, this type of extracellular vesicles derived from CAFs loaded with miRNAs and lncRNAs can be considered as a potential therapeutic target to prevent the development of cancer. Nevertheless, despite the increase in the last 5 years of strong evidence suggesting CAFs-derived exosomal ncRNAs as therapeutic targets, more studies are needed to confirm the protumoral or antitumoral effects exerted by the various miRNAs and lncRNAs. Probably like miRNAs, lncRNAs have unconventional effects on gene expression that are still awaiting to be studied.

Regardless of future studies, the translational way from the laboratory to the clinic has begun. At present, an extensive database of ncRNAs in extracellular vesicles called EVAtlas, a product of enormous work, is available (Liu et al., 2021). In the site http://bioinfo.life.hust.edu.cn/EVAtlas, different characteristics such as extracellular vesicle types and isolation methods, expression level, functions, related drugs, and target genes of seven types of ncRNA of human extracellular vesicles can be consulted, as well as the pathological or physiological condition, tissues, cells, and biological fluids from which they have been isolated. This great bioinformatic tool could be part of the foundations to transfer the findings found in vitro, ex vivo and, in silico on the modulating effects of exosomal ncRNAs, derived and not derived from CAFs in the development of neoplasms, towards therapeutic regimens that improve the prognosis of cancer patients.

Although it is true that it will not be an easy task to incorporate miRNAs and lncRNAs as targets or tools in cancer treatment schemes, their relevance should not be underestimated. In the analysis carried out by GLOBOCAN (Ferlay et al., 2019; Ferlay et al., 2020), the global incidence and mortality rates of different cancers show an increase as the years go by, highlighting the need for additional options than those currently available to help in the fight against neoplasms. Designing and performing safe clinical trials, in which miRNAs and lncRNAs are used as therapeutic tools, is a great challenge and it will be essential to consider them a new real therapeutic alternative that benefits oncological patients.

\section{AUTHOR CONTRIBUTIONS}

JV-P conceptualized and drafted the manuscript. ML-T designed the table and the figure. AR-d-A and AP-S critically reviewed the manuscript. AP-S led the activities. All authors approved the submitted version of the manuscript.

\section{FUNDING}

This work was supported by Sectorial Research Fund for Education, SEP-CONACYT (A1-S-51207), and Jalisco Scientific Development Fund (FODECIJAL) to Attend State Problems 2019 (Project \#8168). JV-P received a CONACYT fellowship (\#769371, second year of Continuity of PostDoctoral Stays Linked to Strengthening the Quality of the National Postgraduate 2020(2), CVU: 377666). 


\section{REFERENCES}

Achreja, A., Zhao, H., Yang, L., Yun, T. H., Marini, J., and Nagrath, D. (2017). ExoMFA - A 13C Metabolic Flux Analysis Framework to Dissect Tumor Microenvironment-Secreted Exosome Contributions Towards Cancer Cell Metabolism. Metab. Eng. 43 (Pt B), 156-172. doi:10.1016/j.ymben.2017.01.001

Admyre, C., Johansson, S. M., Qazi, K. R., Filén, J.-J., Lahesmaa, R., Norman, M., et al. (2007). Exosomes With Immune Modulatory Features Are Present in Human Breast Milk. J. Immunol. 179 (3), 1969-1978. doi:10.4049/ jimmunol.179.3.1969

Ali, S., Suresh, R., Banerjee, S., Bao, B., Xu, Z., Wilson, J., et al. (2015). Contribution of microRNAs in Understanding the Pancreatic Tumor Microenvironment Involving Cancer Associated Stellate and Fibroblast Cells. Am. J. Cancer Res. 5 (3), 1251-1264.

Amit, M., Takahashi, H., Dragomir, M. P., Lindemann, A., Gleber-Netto, F. O., Pickering, C. R., et al. (2020). Loss of P53 Drives Neuron Reprogramming in Head and Neck Cancer. Nature. 578 (7795), 449-454. doi:10.1038/s41586-0201996-3

Baroni, S., Romero-Cordoba, S., Plantamura, I., Dugo, M., D’Ippolito, E., Cataldo, A., et al. (2016). Exosome-Mediated Delivery of miR-9 Induces CancerAssociated Fibroblast-Like Properties in Human Breast Fibroblasts. Cell Death Dis. 7 (7), e2312. doi:10.1038/cddis.2016.224

Beermann, J., Piccoli, M.-T., Viereck, J., and Thum, T. (2016). Non-Coding RNAs in Development and Disease: Background, Mechanisms, and Therapeutic Approaches. Physiol. Rev. 96 (4), 1297-1325. doi:10.1152/physrev.00041.2015

Chen, B., Sang, Y., Song, X., Zhang, D., Wang, L., Zhao, W., et al. (2021). Exosomal miR-500a-5p Derived From Cancer-Associated Fibroblasts Promotes Breast Cancer Cell Proliferation and Metastasis through Targeting USP28. Theranostics. 11 (8), 3932-3947. doi:10.7150/thno.53412

Chen, J.-H., Wu, A. T. H., Bamodu, O. A., Yadav, V. K., Chao, T.-Y., Tzeng, Y.-M., et al. (2019). Ovatodiolide Suppresses Oral Cancer Malignancy by DownRegulating Exosomal Mir-21/stat3/ $\beta$-Catenin Cargo and Preventing Oncogenic Transformation of Normal Gingival Fibroblasts. Cancers. 12, 56. doi:10.3390/ cancers 12010056

Cheng, Q., Li, X., Liu, J., Ye, Q., Chen, Y., Tan, S., et al. (2017). Multiple MyelomaDerived Exosomes Regulate the Functions of Mesenchymal Stem Cells Partially via Modulating miR-21 and miR-146a. Stem Cell Int. 2017, 1-9. doi:10.1155/ $2017 / 9012152$

Dayan, D., Salo, T., Salo, S., Nyberg, P., Nurmenniemi, S., Costea, D. E., et al. (2012). Molecular Crosstalk Between Cancer Cells and Tumor Microenvironment Components Suggests Potential Targets for New Therapeutic Approaches in Mobile Tongue Cancer. Cancer Med. 1 (2), 128-140. doi:10.1002/cam4.24

De Wever, O., Van Bockstal, M., Mareel, M., Hendrix, A., and Bracke, M. (2014). Carcinoma-Associated Fibroblasts Provide Operational Flexibility in Metastasis. Semin. Cancer Biol. 25, 33-46. doi:10.1016/j.semcancer.2013.12.009

Ding, L., Ren, J., Zhang, D., Li, Y., Huang, X., Hu, Q., et al. (2018). A Novel Stromal IncRNA Signature Reprograms Fibroblasts to Promote the Growth of Oral Squamous Cell Carcinoma via LncRNA-CAF/Interleukin-33. Carcinogenesis. 39 (3), 397-406. doi:10.1093/carcin/bgy006

Domvri, K., Petanidis, S., Anestakis, D., Porpodis, K., Bai, C., Zarogoulidis, P., et al. (2020). Exosomal IncRNA PCAT-1 Promotes Kras-Associated Chemoresistance via Immunosuppressive miR-182/miR-217 Signaling and p27/CDK6 Regulation. Oncotarget. 11 (29), 2847-2862. doi:10.18632/ oncotarget. 27675

Donnarumma, E., Fiore, D., Nappa, M., Roscigno, G., Adamo, A., Iaboni, M., et al. (2017). Cancer-associated Fibroblasts Release Exosomal microRNAs That Dictate an Aggressive Phenotype in Breast Cancer. Oncotarget. 8 (12), 19592-19608. doi:10.18632/oncotarget.14752

Dou, D., Ren, X., Han, M., Xu, X., Ge, X., Gu, Y., et al. (2020). Cancer-Associated Fibroblasts-Derived Exosomes Suppress Immune Cell Function in Breast Cancer via the miR-92/pd-L1 Pathway. Front. Immunol. 11, 2026. doi:10.3389/fimmu.2020.02026

Dragomir, M. P., Knutsen, E., and Calin, G. A. (2018). SnapShot: Unconventional miRNA Functions. Cell. 174 (4), 1038. doi:10.1016/j.cell.2018.07.040

Elashiry, M., Elsayed, R., Elashiry, M. M., Rashid, M. H., Ara, R., Arbab, A. S., et al. (2021). Proteomic Characterization, Biodistribution, and Functional Studies of
Immune-Therapeutic Exosomes: Implications for Inflammatory Lung Diseases. Front. Immunol. 12, 636222. doi:10.3389/fimmu.2021.636222

Fang, T., Lv, H., Lv, G., Li, T., Wang, C., Han, Q., et al. (2018). Tumor-Derived Exosomal miR-1247-3p Induces Cancer-Associated Fibroblast Activation to foster Lung Metastasis of Liver Cancer. Nat. Commun. 9 (1), 191. doi:10.1038/ s41467-017-02583-0

Ferlay, J., Colombet, M., Soerjomataram, I., Mathers, C., Parkin, D. M., Piñeros, M., et al. (2019). Estimating the Global Cancer Incidence and Mortality in 2018: GLOBOCAN Sources and Methods. Int. J. Cancer. 144 (8), 1941-1953. doi:10.1002/ijc.31937

Ferlay, J., Laversanne, M., Ervik, M., Lam, F., Colombet, M., Mery, L., et al. (2020). Global Cancer Observatory: Cancer Tomorrow. Lyon, France: International Agency for Research on Cancer. Available at: https://gco.iarc.fr/tomorrow (Accessed 20 May, 2021).

Fiori, M. E., Di Franco, S., Villanova, L., Bianca, P., Stassi, G., and De Maria, R. (2019). Cancer-Associated Fibroblasts as Abettors of Tumor Progression at the Crossroads of EMT and Therapy Resistance. Mol. Cancer. 18, 70. doi:10.1186/s12943-019-0994-2

Gao, Q., Fang, X., Chen, Y., Li, Z., and Wang, M. (2021). Exosomal IncRNA UCA1 From Cancer-Associated Fibroblasts Enhances Chemoresistance in Vulvar Squamous Cell Carcinoma Cells. J. Obstet. Gynaecol. Res. 47 (1), 73-87. doi:10.1111/jog.14418

Gao, Y., Li, X., Zeng, C., Liu, C., Hao, Q., Li, W., et al. (2020). CD63 + CancerAssociated Fibroblasts Confer Tamoxifen Resistance to Breast Cancer Cells Through Exosomal miR-22. Adv. Sci. 7 (21), 2002518. doi:10.1002/ advs. 202002518

Gener Lahav, T., Adler, O., Zait, Y., Shani, O., Amer, M., Doron, H., et al. (2019). Melanoma-Derived Extracellular Vesicles Instigate Proinflammatory Signaling in the Metastatic Microenvironment. Int. J. Cancer. 145 (9), 2521-2534. doi:10.1002/ijc.32521

Goulet, C. R., Champagne, A., Bernard, G., Vandal, D., Chabaud, S., Pouliot, F., et al. (2019). Cancer-Associated Fibroblasts Induce Epithelial-Mesenchymal Transition of Bladder Cancer Cells through Paracrine IL-6 Signalling. BMC Cancer. 19, 137. doi:10.1186/s12885-019-5353-6

Gu, J., Qian, H., Shen, L., Zhang, X., Zhu, W., Huang, L., et al. (2012). Gastric Cancer Exosomes Trigger Differentiation of Umbilical Cord Derived Mesenchymal Stem Cells to Carcinoma-Associated Fibroblasts Through TGF- $\beta /$ Smad Pathway. PLoS One. 7 (12), e52465. doi:10.1371/ journal.pone. 0052465

Guo, H., Ha, C., Dong, H., Yang, Z., Ma, Y., and Ding, Y. (2019). CancerAssociated Fibroblast-Derived Exosomal MicroRNA-98-5p Promotes Cisplatin Resistance in Ovarian Cancer by Targeting CDKN1A. Cancer Cel Int. 19, 347. doi:10.1186/s12935-019-1051-3

Han, K.-Y., Tran, J. A., Chang, J.-H., Azar, D. T., and Zieske, J. D. (2017). Potential Role of Corneal Epithelial Cell-Derived Exosomes in Corneal Wound Healing and Neovascularization. Sci. Rep. 7, 40548. doi:10.1038/srep40548

Herrera, M., Llorens, C., Rodríguez, M., Herrera, A., Ramos, R., Gil, B., et al. (2018). Differential Distribution and Enrichment of Non-Coding RNAs in Exosomes From Normal and Cancer-Associated Fibroblasts in Colorectal Cancer. Mol. Cancer. 17, 114. doi:10.1186/s12943-018-0863-4

$\mathrm{Hu}, \mathrm{T}$., and Hu, J. (2019). Melanoma-derived Exosomes Induce Reprogramming Fibroblasts Into Cancer-Associated Fibroblasts via Gm26809 Delivery. Cell Cycle. 18 (22), 3085-3094. doi:10.1080/15384101.2019.1669380

Huang, T. X., Guan, X. Y., and Fu, L. (2019). Therapeutic Targeting of the Crosstalk Between Cancer-Associated Fibroblasts and Cancer Stem Cells. Am. J. Cancer Res. 9 (9), 1889-1904.

Huang, Y. J., Huang, T. H., Yadav, V. K., Sumitra, M. R., Tzeng, D. T., Wei, P. L., et al. (2020). Preclinical Investigation of Ovatodiolide as a Potential Inhibitor of colon Cancer Stem Cells via Downregulating Sphere-Derived Exosomal $\beta$-Catenin/STAT3/miR-1246 Cargoes. Am. J. Cancer Res. 1010 (128), 46402337-46422354.

Jung, W.-H., Yam, N., Chen, C.-C., Elawad, K., Hu, B., and Chen, Y. (2020). ForceDependent Extracellular Matrix Remodeling by Early-Stage Cancer Cells Alters Diffusion and Induces Carcinoma-Associated Fibroblasts. Biomaterials. 234, 119756. doi:10.1016/j.biomaterials.2020.119756

Kim, J. E., Kim, B. G., Jang, Y., Kang, S., Lee, J. H., and Cho, N. H. (2020). The Stromal Loss of miR-4516 Promotes the FOSL1-dependent Proliferation and Malignancy of Triple Negative Breast Cancer. Cancer Lett. 469, 256-265. doi:10.1016/j.canlet.2019.10.039 
Lee, J.-C., Wu, A. T. H., Chen, J.-H., Huang, W.-Y., Lawal, B., Mokgautsi, N., et al. (2020). HNC0014, a Multi-Targeted Small-Molecule, Inhibits Head and Neck Squamous Cell Carcinoma by Suppressing C-Met/STAT3/CD44/PD-L1 Oncoimmune Signature and Eliciting Antitumor Immune Responses. Cancers. 12, 3759. doi:10.3390/cancers12123759

Li, B. L., Lu, W., Qu, J. J., Ye, L., Du, G. Q., and Wan, X. P. (2019). Loss of Exosomal miR-148b From Cancer-Associated Fibroblasts Promotes Endometrial Cancer Cell Invasion and Cancer Metastasis. J. Cel Physiol. 234 (3), 2943-2953. doi:10.1002/jcp. 27111

Li, K., Liu, T., Chen, J., Ni, H., and Li, W. (2020a). Survivin in Breast CancerDerived Exosomes Activates Fibroblasts by Up-Regulating SOD1, Whose Feedback Promotes Cancer Proliferation and Metastasis. J. Biol. Chem. 295 (40), 13737-13752. doi:10.1074/jbc.RA120.013805

Li, Y., Zhao, Z., Liu, W., and Li, X. (2020b). SNHG3 Functions as miRNA Sponge to Promote Breast Cancer Cells Growth Through the Metabolic Reprogramming. Appl. Biochem. Biotechnol. 191 (3), 1084-1099. doi:10.1007/s12010-02003244-7

Li, W., Zhang, X., Wang, J., Li, M., Cao, C., Tan, J., et al. (2017). TGF $\beta 1$ in Fibroblasts-Derived Exosomes Promotes Epithelial-Mesenchymal Transition of Ovarian Cancer Cells. Oncotarget. 8 (56), 96035-96047. doi:10.18632/ oncotarget. 21635

Li, Y.-y., Tao, Y.-w., Gao, S., Li, P., Zheng, J.-m., Zhang, S.-e., et al. (2018). CancerAssociated Fibroblasts Contribute to Oral Cancer Cells Proliferation and Metastasis via Exosome-Mediated Paracrine miR-34a-5p. EBioMedicine. 36, 209-220. doi:10.1016/j.ebiom.2018.09.006

Liu, C.-J., Xie, G.-Y., Miao, Y.-R., Xia, M., Wang, Y., Lei, Q., et al. (2021). EVAtlas: a Comprehensive Database for ncRNA Expression in Human Extracellular Vesicles. Nucleic Acids Res., 1-7. doi:10.1093/nar/gkab668

Liu, L., Zhang, Z., Zhou, L., Hu, L., Yin, C., Qing, D., et al. (2020). Cancer Associated Fibroblasts-Derived Exosomes Contribute to Radioresistance Through Promoting Colorectal Cancer Stem Cells Phenotype. Exp. Cel Res. 391 (2), 111956. doi:10.1016/j.yexcr.2020.111956

Luga, V., and Wrana, J. L. (2013). Tumor-Stroma Interaction: Revealing Fibroblast-Secreted Exosomes as Potent Regulators of Wnt-Planar Cell Polarity Signaling in Cancer Metastasis. Cancer Res. 73 (23), 6843-6847. doi:10.1158/0008-5472.CAN-13-1791

Lv, B., Zhu, W., and Feng, C. (2020). Coptisine Blocks Secretion of Exosomal Circcet3 From Cancer-Associated Fibroblasts to Reprogram Glucose Metabolism in Hepatocellular Carcinoma. DNA Cel Biol. 39 (12), 2281-2288. doi:10.1089/dna.2020.6058

Mashouri, L., Yousefi, H., Aref, A. R., Ahadi, A. m., Molaei, F., and Alahari, S. K. (2019). Exosomes: Composition, Biogenesis, and Mechanisms in Cancer Metastasis and Drug Resistance. Mol. Cancer. 18, 75. doi:10.1186/s12943019-0991-5

Masoud, G. N., and Li, W. (2015). HIF-1a Pathway: Role, Regulation and Intervention for Cancer Therapy. Acta Pharmaceutica Sinica B. 5 (5), 378-389. doi:10.1016/j.apsb.2015.05.007

Mathivanan, S., Ji, H., and Simpson, R. J. (2010). Exosomes: Extracellular Organelles Important in Intercellular Communication. J. Proteomics. 73 (10), 1907-1920. doi:10.1016/j.jprot.2010.06.006

Nilsson, J., Skog, J., Nordstrand, A., Baranov, V., Mincheva-Nilsson, L., Breakefield, X. O., et al. (2009). Prostate Cancer-Derived Urine Exosomes: a Novel Approach to Biomarkers for Prostate Cancer. Br. J. Cancer. 100 (10), 1603-1607. doi:10.1038/sj.bjc.6605058

Nouraee, N., Khazaei, S., Vasei, M., Razavipour, S. F., Sadeghizadeh, M., and Mowla, S. J. (2016). MicroRNAs Contribution in Tumor Microenvironment of Esophageal Cancer. Cbm. 16 (3), 367-376. doi:10.3233/CBM-160575

Pathania, R., Ramachandran, S., Elangovan, S., Padia, R., Yang, P., Cinghu, S., et al. (2015). DNMT1 Is Essential for Mammary and Cancer Stem Cell Maintenance and Tumorigenesis. Nat. Commun. 6, 6910. doi:10.1038/ncomms7910

Principe, S., Mejia-Guerrero, S., Ignatchenko, V., Sinha, A., Ignatchenko, A., Shi, W., et al. (2018). Proteomic Analysis of Cancer-Associated Fibroblasts Reveals a Paracrine Role for MFAP5 in Human Oral Tongue Squamous Cell Carcinoma. J. Proteome Res. 17 (6), 2045-2059. doi:10.1021/acs.jproteome.7b00925

Qin, X., Guo, H., Wang, X., Zhu, X., Yan, M., Wang, X., et al. (2019). Exosomal miR-196a Derived from Cancer-Associated Fibroblasts Confers Cisplatin Resistance in Head and Neck Cancer Through Targeting CDKN1B and ING5. Genome Biol. 20, 12. doi:10.1186/s13059-018-1604-0
Ramteke, A., Ting, H., Agarwal, C., Mateen, S., Somasagara, R., Hussain, A., et al. (2015). Exosomes Secreted under Hypoxia Enhance Invasiveness and Stemness of Prostate Cancer Cells by Targeting Adherens junction Molecules. Mol. Carcinog. 54 (7), 554-565. doi:10.1002/mc.22124

Ren, J., Ding, L., Zhang, D., Shi, G., Xu, Q., Shen, S., et al. (2018). CarcinomaAssociated Fibroblasts Promote the Stemness and Chemoresistance of Colorectal Cancer by Transferring Exosomal lncRNA H19. Theranostics. 8 (14), 3932-3948. doi:10.7150/thno.25541

Richards, K. E., Zeleniak, A. E., Fishel, M. L., Wu, J., Littlepage, L. E., and Hill, R. (2017). Cancer-Associated Fibroblast Exosomes Regulate Survival and Proliferation of Pancreatic Cancer Cells. Oncogene. 36 (13), 1770-1778. doi:10.1038/onc.2016.353

Ringuette Goulet, C., Bernard, G., Tremblay, S., Chabaud, S., Bolduc, S., and Pouliot, F. (2018). Exosomes Induce Fibroblast Differentiation Into CancerAssociated Fibroblasts Through TGF $\beta$ Signaling. Mol. Cancer Res. 16 (7), 1196-1204. doi:10.1158/1541-7786.MCR-17-0784

Sansone, P., Savini, C., Kurelac, I., Chang, Q., Amato, L. B., Strillacci, A., et al. (2017). Packaging and Transfer of Mitochondrial DNA via Exosomes Regulate Escape From Dormancy in Hormonal Therapy-Resistant Breast Cancer. Proc. Natl. Acad. Sci. USA. 114 (43), E9066-E9075. doi:10.1073/pnas.1704862114

Seo, N., Akiyoshi, K., and Shiku, H. (2018). Exosome-Mediated Regulation of Tumor Immunology. Cancer Sci. 109 (10), 2998-3004. doi:10.1111/cas.13735

Shah, S. H., Miller, P., Garcia-Contreras, M., Ao, Z., Machlin, L., Issa, E., et al. (2015). Hierarchical Paracrine Interaction of Breast Cancer Associated Fibroblasts With Cancer Cells via hMAPK-microRNAs to Drive ERNegative Breast Cancer Phenotype. Cancer Biol. Ther. 16 (11), 1671-1681. doi:10.1080/15384047.2015.1071742

Shan, G., Gu, J., Zhou, D., Li, L., Cheng, W., Wang, Y., et al. (2020). CancerAssociated Fibroblast-Secreted Exosomal miR-423-5p Promotes Chemotherapy Resistance in Prostate Cancer by Targeting GREM2 Through the TGF- $\beta$ Signaling Pathway. Exp. Mol. Med. 52 (11), 1809-1822. doi:10.1038/ s12276-020-0431-z

Shan, G., Zhou, X., Gu, J., Zhou, D., Cheng, W., Wu, H., et al. (2021). Downregulated Exosomal microRNA-148b-3p in Cancer Associated Fibroblasts Enhance Chemosensitivity of Bladder Cancer Cells by Downregulating the Wnt $/ \beta$-Catenin Pathway and Upregulating PTEN. Cell Oncol. 44, 45-59. doi:10.1007/s13402-020-00500-0

Shimoda, M., Principe, S., Jackson, H. W., Luga, V., Fang, H., Molyneux, S. D., et al. (2014). Loss of the Timp Gene Family Is Sufficient for the Acquisition of the CAF-Like Cell State. Nat. Cel Biol. 16 (9), 889-901. doi:10.1038/ncb3021

Sui, C., Meng, F., Li, Y., and Jiang, Y. (2015). miR-148b Reverses CisplatinResistance in Non-Small Cell Cancer Cells via Negatively Regulating DNA (Cytosine-5)-Methyltransferase 1(DNMT1) Expression. J. Transl. Med. 13, 132. doi:10.1186/s12967-015-0488-y

Sun, L. P., Xu, K., Cui, J., Yuan, D. Y., Zou, B., Li, J., et al. (2019). Cancer associated Fibroblast derived Exosomal miR3825p Promotes the Migration and Invasion of Oral Squamous Cell Carcinoma. Oncol. Rep. 42 (4), 1319-1328. doi:10.3892/ or.2019.7255

Sun, W., and Fu, S. (2019). Role of Cancer-Associated Fibroblasts in Tumor Structure, Composition and the Microenvironment in Ovarian Cancer (Review). Oncol. Lett. 18 (3), 2173-2178. doi:10.3892/ol.2019.10587

Théry, C. (2011). Exosomes: Secreted Vesicles and Intercellular Communications. F1000 Biol. Rep. 3, 15. doi:10.3410/B3-15

Vered, M., Lehtonen, M., Hotakainen, L., Pirilä, E., Teppo, S., Nyberg, P., et al. (2015). Caveolin-1 Accumulation in the Tongue Cancer Tumor Microenvironment Is Significantly Associated with Poor Prognosis: an InVivo and In-Vitro Study. BMC Cancer. 15, 25. doi:10.1186/s12885-015-1030-6

Wang, H., Wei, H., Wang, J., Li, L., Chen, A., and Li, Z. (2020). MicroRNA-181d5p-Containing Exosomes Derived From CAFs Promote EMT by Regulating CDX2/HOXA5 in Breast Cancer. Mol. Ther. - Nucleic Acids. 19, 654-667. doi:10.1016/j.omtn.2019.11.024

Wang, J.-W., Wu, X.-F., Gu, X.-J., and Jiang, X.-H. (2019a). Exosomal miR-1228 From Cancer-Associated Fibroblasts Promotes Cell Migration and Invasion of Osteosarcoma by Directly Targeting SCAI. Oncol. Res. 27 (9), 979-986. doi:10.3727/096504018X15336368805108

Wang, X., Qin, X., Yan, M., Shi, J., Xu, Q., Li, Z., et al. (2019b). Loss of Exosomal miR-3188 in Cancer-Associated Fibroblasts Contributes to HNC Progression. J. Exp. Clin. Cancer Res. 38, 151. doi:10.1186/s13046-019-1144-9 
Wu, H.-J., Hao, M., Yeo, S. K., and Guan, J.-L. (2020). FAK Signaling in CancerAssociated Fibroblasts Promotes Breast Cancer Cell Migration and Metastasis by Exosomal miRNAs-Mediated Intercellular Communication. Oncogene. 39 (12), 2539-2549. doi:10.1038/s41388-020-1162-2

Yang, Y., Li, J., and Geng, Y. (2020). Exosomes Derived from Chronic Lymphocytic Leukaemia Cells Transfer miR-146a to Induce the Transition of Mesenchymal Stromal Cells into Cancer-Associated Fibroblasts. J. Biochem. 168 (5), 491-498. doi:10.1093/jb/mvaa064

Yeon, J. H., Jeong, H. E., Seo, H., Cho, S., Kim, K., Na, D., et al. (2018). CancerDerived Exosomes Trigger Endothelial to Mesenchymal Transition Followed by the Induction of Cancer-Associated Fibroblasts. Acta Biomater. 76, 146-153. doi:10.1016/j.actbio.2018.07.001

You, J., Li, M., Cao, L. M., Gu, Q. H., Deng, P. B., Tan, Y., et al. (2019). SnaillDependent Cancer-Associated Fibroblasts Induce Epithelial-Mesenchymal Transition in Lung Cancer Cells via Exosomes. QJM. 112 (8), 581-590. doi:10.1093/qjmed/hcz093

Zhang, H., Deng, T., Liu, R., Ning, T., Yang, H., Liu, D., et al. (2020a). CAF Secreted miR-522 Suppresses Ferroptosis and Promotes Acquired Chemo-Resistance in Gastric Cancer. Mol. Cancer. 19, 43. doi:10.1186/s12943-020-01168-8

Zhang, N., Wang, Y., Liu, H., and Shen, W. (2020b). Extracellular Vesicle Encapsulated microRNA-320a Inhibits Endometrial Cancer by Suppression of the HIF1 $\alpha$ /VEGFA axis. Exp. Cel Res. 394 (2), 112113. doi:10.1016/j.yexcr.2020.112113

Zhang, H. W., Shi, Y., Liu, J. B., Wang, H. M., Wang, P. Y., Wu, Z. J., et al. (2021). Cancer-associated Fibroblast-Derived Exosomal microRNA-24-3p Enhances Colon Cancer Cell Resistance to MTX by Down-Regulating CDX2/HEPH axis. J. Cel Mol. Med. 25 (8), 3699-3713. doi:10.1111/jcmm.15765

Zhang, Y.-F., Zhou, Y.-Z., Zhang, B., Huang, S.-F., Li, P.-P., He, X.-M., et al. (2019a). Pancreatic Cancer-Derived Exosomes Promoted Pancreatic Stellate Cells Recruitment by Pancreatic Cancer. J. Cancer. 10 (18), 4397-4407. doi:10.7150/jca.27590

Zhang, Y., Cai, H., Chen, S., Sun, D., Zhang, D., and He, Y. (2019b). Exosomal Transfer of miR-124 Inhibits normal Fibroblasts to Cancer-Associated Fibroblasts Transition by Targeting Sphingosine Kinase 1 in Ovarian Cancer. J. Cel Biochem. 120 (8), 13187-13201. doi:10.1002/jcb.28593

Zhang, Z., Li, X., Sun, W., Yue, S., Yang, J., Li, J., et al. (2017). Loss of Exosomal miR-320a From Cancer-Associated Fibroblasts Contributes to HCC
Proliferation and Metastasis. Cancer Lett. 397, 33-42. doi:10.1016/ j.canlet.2017.03.004

Zhao, G., Li, H., Guo, Q., Zhou, A., Wang, X., Li, P., et al. (2020). Exosomal Sonic Hedgehog Derived From Cancer-Associated Fibroblasts Promotes Proliferation and Migration of Esophageal Squamous Cell Carcinoma. Cancer Med. 9 (7), 2500-2513. doi:10.1002/cam4.2873

Zhao, H., Yang, L., Baddour, J., Achreja, A., Bernard, V., Moss, T., et al. (2016). Tumor Microenvironment Derived Exosomes Pleiotropically Modulate Cancer Cell Metabolism. Elife. 5, e10250. doi:10.7554/eLife.10250

Zhao, Q., Huang, L., Qin, G., Qiao, Y., Ren, F., Shen, C., et al. (2021). Cancerassociated Fibroblasts Induce Monocytic Myeloid-Derived Suppressor Cell Generation via IL-6/Exosomal miR-21-Activated STAT3 Signaling to Promote Cisplatin Resistance in Esophageal Squamous Cell Carcinoma. Cancer Lett. 518, 35-48. doi:10.1016/j.canlet.2021.06.009

Zhou, L., Li, J., Tang, Y., and Yang, M. (2021). Exosomal LncRNA LINC00659 Transferred From Cancer-Associated Fibroblasts Promotes Colorectal Cancer Cell Progression via miR-342-3p/ANXA2 axis. J. Transl. Med. 19, 8. doi:10.1186/s12967-020-02648-7

Conflict of Interest: The authors declare that the research was conducted in the absence of any commercial or financial relationships that could be construed as a potential conflict of interest.

Publisher's Note: All claims expressed in this article are solely those of the authors and do not necessarily represent those of their affiliated organizations, or those of the publisher, the editors and the reviewers. Any product that may be evaluated in this article, or claim that may be made by its manufacturer, is not guaranteed or endorsed by the publisher.

Copyright (c) 2021 Villegas-Pineda, Lizarazo-Taborda, Ramírez-de-Arellano and Pereira-Suárez. This is an open-access article distributed under the terms of the Creative Commons Attribution License (CC BY). The use, distribution or reproduction in other forums is permitted, provided the original author(s) and the copyright owner(s) are credited and that the original publication in this journal is cited, in accordance with accepted academic practice. No use, distribution or reproduction is permitted which does not comply with these terms. 